\title{
Effect of Chronic Phenelzine Treatment on REM Sleep: Report of Three Patients
}

\author{
Hans-Peter Landolt, Ph.D. and Lieselotte Posthuma de Boer, M.S.
}

\begin{abstract}
Antidepressants belonging to the class of monoamine oxidase inhibitors (MAOI) such as phenelzine have long been known to drastically suppress REM sleep. Sleep and the electroencephalogram (EEG) in sleep and waking were studied in three depressed patients at regular time intervals before, during and after 6 to 18 months of phenelzine treatment. While REM sleep was initially eliminated in all patients, short REM sleep episodes reappeared after three to six months of medication. Total sleep time and EEG slow-wave activity (SWA, spectral power within $0.75-4.5 \mathrm{~Hz}$ ) in nonREM sleep
\end{abstract}

(stages 1-4) were not changed. In contrast, EEG theta frequency activity (TFA, power within 4.75-8.0 Hz) during a 5-min wake interval recorded prior to the sleep episodes was initially enhanced, and tended to correlate negatively with the percentage of REM sleep $(p=.06)$. This observation indicates that compensatory REM sleep mechanisms may occur in wakefulness during chronic MAOI treatment.

[Neuropsychopharmacology 25:S63-S67, 2001]

(C) 2001 American College of Neuropsychopharmacology.

Published by Elsevier Science Inc.
KEY WORDS: Monoamine oxidase inhibitor (MAOI); Sleep EEG; Wakefulness; Slow-wave activity; Theta activity

The monoamine oxidase inhibitor (MAOI) phenelzine is an effective "first generation" antidepressant capable of virtually abolishing rapid-eye-movement (REM) sleep at a therapeutic dose range (Akindele et al. 1970; Wyatt et al. 1971a,b; Dunleavy and Oswald 1973; Landolt et al. 2001). Although early studies suggested that REM sleep deprivation was psychologically harmful (Dement 1960), a few patients with depression or narcolepsy have been treated safely and successfully with phenelzine for prolonged periods of time (Wyatt et al. 1971a,b). While complete REM sleep suppression for as long as seven months has been described, no study has so far investigated the effects of the drug on sleep and the sleep electroencephalogram (EEG) for longer treatment periods. To examine whether chronic

From the University of California at San Diego, Department of Psychiatry, VA San Diego Healthcare System, San Diego, California, USA

Address correspondence to: Hans-Peter Landolt, Ph.D., Institute of Pharmacology and Toxicology, University of Zürich, Winterthurerstrasse,190, 8057 Zürich, Switzerland, Tel.: +41-1-635-5953, Fax: +41-1-635-5707, E-mail: landolt@pharma.unizh.ch
MAOI administration is associated with persistent absence of REM sleep, we studied sleep and the EEG in sleep and waking in three depressed patients at regular time intervals before, during and after 6 to 18 months of phenelzine treatment.

\section{METHODS}

\section{Patients}

Patient PM. This 35-year-old woman suffered from major depressive disorder (MDD) with loss of interest or pleasure, insomnia, feelings of worthlessness, diminished ability to concentrate, recurrent thoughts of death, and suicidal ideation. She also met criteria for generalized anxiety disorder. Structured clinical psychiatric diagnostic interview according to DSM-IV (SCID) revealed a history of two previous episodes of major depression, which were treated with psychotherapy and fluoxetine. The patient denied a history of manic/hypomanic episodes and abuse of alcohol or drugs. Her mother and a maternal uncle suffered from bipolar disorder.

Patient RH. The SCID diagnosis of this 37-year old man consisted of MDD, single episode. His symptoms 
were severe and included depressed mood, loss of interest or pleasure, psychomotor retardation, lack of energy, worthlessness, difficulty thinking or concentrating, and suicidal ideation. Mood disturbances started at age 10 and met formal criteria of MDD since age 15; they were never treated. Secondary SCID diagnoses included alcohol, cannabis and cocaine dependence in sustained full remission. The family history was negative for psychiatric disorders.

Patient CS. The SCID of this 56-year old man revealed the single diagnosis of MDD with depressed mood, lack of interest or pleasure, insomnia, psychomotor retardation, feelings of worthlessness, and diminished ability to think or concentrate. Three previous major depressive episodes at ages 20,48, and 51 were treated with trazodone, fluoxetine and buspirone, respectively. The patient had no family history of psychiatric disorders.

\section{Study Protocol, Phenelzine Treatment and Polygraphic Recordings}

The study protocol was approved by the local Institutional Review Board (IRB), and written informed consent was obtained prior to the study. The patients were seen weekly for clinical visits (bi-weekly after remission). They were instructed to keep a regular sleepwake cycle and to avoid daytime naps. A total of 60 nocturnal sleep episodes scheduled at their habitual bedtimes were recorded in the sleep laboratory during a drug-free baseline night prior to initiation of antidepressant therapy, at 2 to 4 -week intervals during phenelzine treatment, and at 3 to 7 -day intervals during dose tapering and withdrawal (patients $\mathrm{PM}$ and $\mathrm{RH}$ ). The data of two recordings (one in $\mathrm{RH}$ and one in CS) were lost because of technical difficulties.

In all patients, open-labeled treatment with the MAOI phenelzine was initiated after the baseline night, and individual daily doses of $15-75 \mathrm{mg}$ were prescribed according to clinical considerations (Table 1). To verify compliance, the patients were instructed to check off a box in a drug diary after medication intake. The treatment period was arbitrarily subdivided into a subchronic (1-18 weeks) and chronic ( $>18$ weeks) phase.

Nine EEGs (the data of the C3-A2 derivation are reported here), submental EMG, bipolar EOG and ECG were recorded by a portable amplifier system during a 5-min wake interval prior to lights-out (starting at 10:05 P.M. \pm 20 min [SD] in PM, 10:23 P.M. $\pm 23 \mathrm{~min}$ in RH, and 10:27 P.M. $\pm 42 \mathrm{~min}$ in CS), and the entire sleep episode. The detailed procedures of data recording and signal conditioning have been described previously (Landolt et al. 2001). Each recording night was preceded by at least one adaptation night. The sleep stages were visually scored according to Rechtschaffen and
Table 1. Schedule of Phenelzine Treatment

\begin{tabular}{|c|c|c|c|c|c|c|}
\hline \multirow{2}{*}{$\begin{array}{l}\text { Phase of } \\
\text { treatment }\end{array}$} & \multicolumn{2}{|c|}{ Patient PM } & \multicolumn{2}{|c|}{ Patient RH } & \multicolumn{2}{|c|}{ Patient CS } \\
\hline & Week & mg/day & Week & mg/day & Week & mg/day \\
\hline Baseline & -1 & - & -1 & - & -4 & - \\
\hline Subchronic & $\begin{array}{c}1 \\
2-3 \\
4-5 \\
6-18\end{array}$ & $\begin{array}{c}15-45 \\
30 \\
45 \\
60\end{array}$ & $\begin{array}{c}1 \\
2 \\
3-18\end{array}$ & $\begin{array}{c}15-45 \\
60 \\
75\end{array}$ & $\begin{array}{c}1 \\
2 \\
3-18\end{array}$ & $\begin{array}{c}15-30 \\
45 \\
60\end{array}$ \\
\hline Chronic & $\begin{array}{l}19-23 \\
24-30\end{array}$ & $\begin{array}{l}60 \\
75\end{array}$ & $19-75$ & 75 & $\begin{array}{c}19-21 \\
>22\end{array}$ & $\begin{array}{l}60 \\
45\end{array}$ \\
\hline $\begin{array}{l}\text { Tapering/ } \\
\text { Withdrawal }\end{array}$ & $\begin{array}{l}31 \\
32\end{array}$ & $\begin{array}{c}45-15 \\
-\end{array}$ & $\begin{array}{c}76-79 \\
80\end{array}$ & $\begin{array}{c}60-15 \\
-\end{array}$ & & \\
\hline
\end{tabular}

Week: week of treatment; $\mathrm{mg}$ /day: daily dose of phenelzine.

Kales (1968), and the EEG was subjected to power spectral analysis (Landolt et al. 2001).

\section{RESULTS}

\section{Treatment Response and Visually Scored Sleep Variables}

All patients remitted from depression by week 5 on phenelzine (HRSD and BDI scores $\leqslant 10$; Table 2). The treatment response was especially impressive in $\mathrm{RH}$, who had been severely depressed for 20 years prior to the study.

The sleep profiles and the time course of EEG slowwave activity (SWA, power within $0.75-4.5 \mathrm{~Hz}$ ) of representative nights of $\mathrm{PM}$ and $\mathrm{RH}$ in all treatment phases are illustrated in Figure 1. REM sleep was completely eliminated in all patients by day 43 of pharmacotherapy. In PM, REM sleep was essentially absent for six months except for short REM sleep episodes (range: 4-28 min) during the fifth month on phenelzine (Figure 1). REM sleep disappeared again after increasing the dose of the drug. In RH, REM sleep was initially eliminated for five months. Various amounts of REM sleep (range: 9-134 $\mathrm{min}$ ) were detected in most sleep recordings during the remainder of pharmacotherapy. In CS, REM sleep was absent for three months. Thereafter, REM sleep episodes of various durations (range: $1-53 \mathrm{~min}$ ) reappeared in most sleep recordings. The suppression of REM sleep was accompanied by increased amount of stage 2 (PM), slow wave sleep (stages $3 \& 4$ ) and/or wakefulness after sleep onset (WASO; RH and CS). Total sleep time (TST) was not affected (Table 2).

Phenelzine treatment in PM was stopped because of weight gain and edema. Tapering the dose gave rise to an impressive REM sleep rebound, for example a 2-h sleep onset REM sleep period and more than three hours of REM sleep ( $46 \%$ of TST) in the third 
Table 2. Visually Scored Sleep Variables, Theta Frequency Activity in Waking, and Mood Ratings (Means \pm SD)

\begin{tabular}{|c|c|c|c|c|c|c|c|c|}
\hline & HRSD & BDI & $\begin{array}{c}\text { REM sleep } \\
(\%)\end{array}$ & Stage $2(\%)$ & SWS (\%) & $\begin{array}{c}\text { WASO } \\
(\%)\end{array}$ & TST (min) & $\begin{array}{c}\text { TFA } \\
\left(\mu \mathbf{V}^{2} / \mathrm{Hz}\right)\end{array}$ \\
\hline \multicolumn{9}{|l|}{ Patient PM } \\
\hline Baseline & 22 & 22 & 12.4 & 53.7 & 23.8 & 17.2 & 396.3 & 40.9 \\
\hline Subchronic $(n=8)$ & $3.7 \pm 2.8$ & $3.0 \pm 3.1$ & $2.1 \pm 5.5$ & $74.2 \pm 11.3$ & $18.1 \pm 5.5$ & $10.4 \pm 6.3$ & $405.5 \pm 34.9$ & $135.2 \pm 71.9$ \\
\hline Chronic $(n=7)$ & $4.3 \pm 1.6$ & $0.5 \pm .05$ & $1.9 \pm 2.6$ & $78.3 \pm 4.3$ & $15.0 \pm 4.9$ & $4.1 \pm 3.3$ & $397.2 \pm 21.8$ & $121.9 \pm 25.2$ \\
\hline Withdrawal $(n=6)$ & $7.3 \pm 2.5$ & $2.3 \pm 0.6$ & $25.6 \pm 16.6$ & $45.1 \pm 18.3$ & $17.6 \pm 2.8$ & $7.8 \pm 4.2$ & $378.9 \pm 17.1$ & $52.5 \pm 20.6$ \\
\hline $\mathrm{F}_{3,21}(\mathrm{p})$ & $19.0(<0.001)$ & $29.8(<0.001)$ & $8.8(<0.001)$ & $9.9(<0.001)$ & 1.3 (n.s.) & $3.4(0.04)$ & 1.2 (n.s.) & $4.0(0.02)$ \\
\hline \multicolumn{9}{|l|}{ Patient RH } \\
\hline Baseline & 28 & 36 & 14.4 & 52.5 & 24.5 & 14.5 & 373.0 & 53.8 \\
\hline Subchronic $(n=7)$ & $6.3 \pm 6.2$ & $7.6 \pm 9.5$ & $0.1 \pm 0.4$ & $52.9 \pm 14.2$ & $40.4 \pm 15.7$ & $23.8 \pm 13.6$ & $343.2 \pm 46.8$ & $86.0 \pm 35.3$ \\
\hline Chronic $(n=12)$ & $0.7 \pm 0.9$ & $0.1 \pm 0.3$ & $6.9 \pm 10.3$ & $50.7 \pm 11.0$ & $33.7 \pm 11.7$ & $17.3 \pm 10.1$ & $382.6 \pm 47.4$ & $51.0 \pm 28.7$ \\
\hline Withdrawal $(n=6)$ & $0.0 \pm 0.0$ & $0.0 \pm 0.0$ & $26.7 \pm 5.8$ & $48.8 \pm 6.8$ & $15.7 \pm 7.4$ & $5.0 \pm 1.6$ & $446.2 \pm 32.9$ & $40.4 \pm 22.5$ \\
\hline $\mathrm{F}_{3,25}(\mathrm{p})$ & $21.2(<0.001)$ & $15.7(<0.001)$ & $13.6(<0.001)$ & 0.2 (n.s.) & $4.8(0.01)$ & $3.8(0.03)$ & $5.9(0.004)$ & $3.1(0.05)$ \\
\hline \multicolumn{9}{|c|}{ 20.5 ( } \\
\hline Baseline & 23 & 27 & 21. & 60 & 4.0 & 13.4 & 310.7 & 20.4 \\
\hline Subchronic $(n=4)$ & $5.6 \pm 3.2$ & $2.6 \pm 2.7$ & $3.4 \pm 3.9$ & $73.3 \pm 9.6$ & $9.1 \pm 10.8$ & $30.4 \pm 19.4$ & $342.7 \pm 40.2$ & $41.4 \pm 12.8$ \\
\hline Chronic $(n=5)$ & $5.6 \pm 1.5$ & $2.2 \pm 0.4$ & $3.5 \pm 7.2$ & $69.6 \pm 6.0$ & $3.9 \pm 3.8$ & $56.8 \pm 12.0$ & $310.2 \pm 23.7$ & $26.0 \pm 12.9$ \\
\hline $\mathrm{F}_{2,9}(\mathrm{p})$ & $21.8(<0.001)$ & $73.4(<0.001)$ & $4.1(0.07)$ & 1.1 (n.s.) & 0.6 (n.s.) & $5.0(0.04)$ & 1.2 (n.s.) & 2.2 (n.s.) \\
\hline
\end{tabular}

HRSD: Hamilton Rating Scale of Depression (24 items). BDI: Beck Depression Inventory. The sleep variables are given in minutes (TST, total sleep time) or as a percentage of TST. SWS: slow wave sleep. WASO: waking after sleep onset. TFA: theta frequency activity (power within $4.75-8.0 \mathrm{~Hz}$ ) in a 5 -min wake period prior to sleep.

F and $p$ values: One-way ANOVA with factor 'treatment phase' (baseline, subchronic, chronic, withdrawal). n.s.: no significant change.

night after withdrawal. A REM sleep rebound occurred also in RH. It was most prominent after one week of dose tapering, with 162 min of REM sleep (33\% of TST). Elevated REM sleep levels ( $>30 \%$ ) were observed in both patients for approximately two weeks after the reduction of phenelzine. In CS, the dose of medication was reduced after six months due to complaints of drowsiness and sexual side effects. At present, he is still in treatment, but non-compliance with the dosing schedule and non-availability of the PS-1 recording system precluded further sleep studies.

\section{EEG Power Spectra in NonREM Sleep and Waking}

Phenelzine treatment had no effect on nonREM sleep (stages 1-4) intensity as quantified by EEG SWA $\left(\mathrm{F}_{3,57}=\right.$ $1.1, p>.3$ ). Similarly, theta frequency activity (TFA, power within $4.75-8.0 \mathrm{~Hz}$ ) in nonREM sleep was not changed $\left(\mathrm{F}_{3,57}=1.0, p>.4\right)$.

Statistical analysis of TFA during the 5-min wake interval prior to the sleep episodes revealed a significant effect of 'treatment phase', with the highest value present during subchronic phenelzine administration (Table 2; $\mathrm{F}_{3,57}=3.4, p<.03$ ). Spearman rank correlation analysis between the mean percentage of REM sleep and TFA revealed a trend toward a negative correlation between these two variables $\left(\mathrm{r}_{\mathrm{s}}=-0.58, p=.06, n=11\right)$.

\section{DISCUSSION}

The exact mechanism underlying MAOI-induced suppression of REM sleep is unknown. Previous studies in- vestigating the effect of phenelzine have suggested that REM sleep in humans can be drastically suppressed, or even completely eliminated, for weeks to months without obvious adverse effects (Akindele et al. 1970; Wyatt et al. 1971a,b; Dunleavy and Oswald 1973; Landolt et al. 2001). As pointed out by Horne (2000), these findings might indicate that (1) the drug substitutes for REM sleep, (2) the medication affects REM sleep control mechanisms rather than REM sleep function, or (3) that REM sleep has no unique benefit that cannot be met by either nonREM sleep or wakefulness.

We found in a small sample of depressed patients who were chronically and successfully treated with phenelzine that the drug eliminated REM sleep for three to six months. The loss of REM sleep was associated with increased duration of stage 2 or SWS, whereas TST and EEG SWA were not changed. During continued treatment, episodes of REM sleep reappeared in all patients. They occurred at regular time intervals during sleep, and coincided roughly with REM sleep periods in the baseline recordings (Figure 1). This observation indicates that the drug is not sufficient to substitute for REM sleep.

Central nervous mechanisms of adaptation or tolerance may have allowed REM sleep to reoccur after several months of MAOI treatment. The amount of REM sleep, however, was still reduced during the chronic phase of medication when compared with baseline (Table 2). Complete REM sleep disinhibition occurred only in the phase of treatment where the drug was reduced. It was followed by two weeks of REM sleep rebound, which was observed in both patients who were studied during dose tapering and withdrawal. This finding in- 


\section{Patient PM}

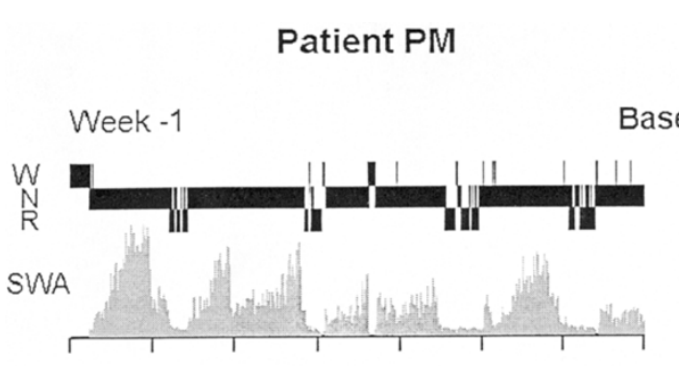

Baseline

Patient RH

Week 12

Phenelzine treatment, subchronic

Week 19
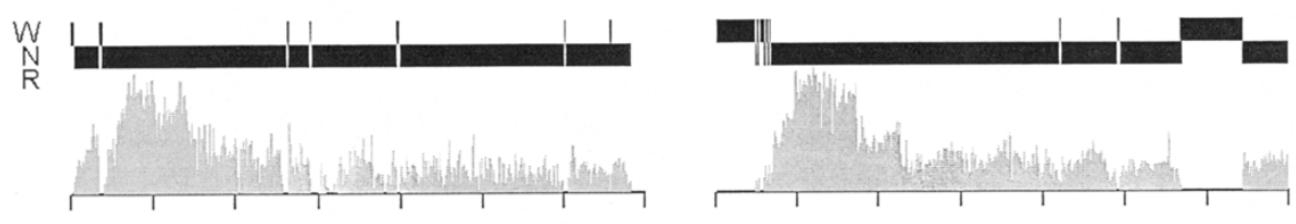

Week 23

Phenelzine treatment, chronic

Week 49
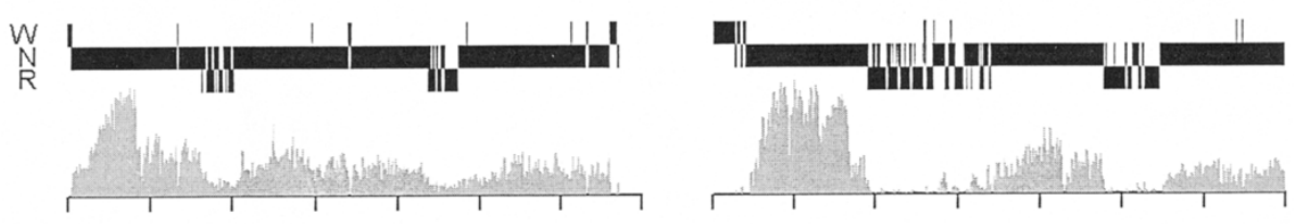

Week 32

Withdrawal

Week 80
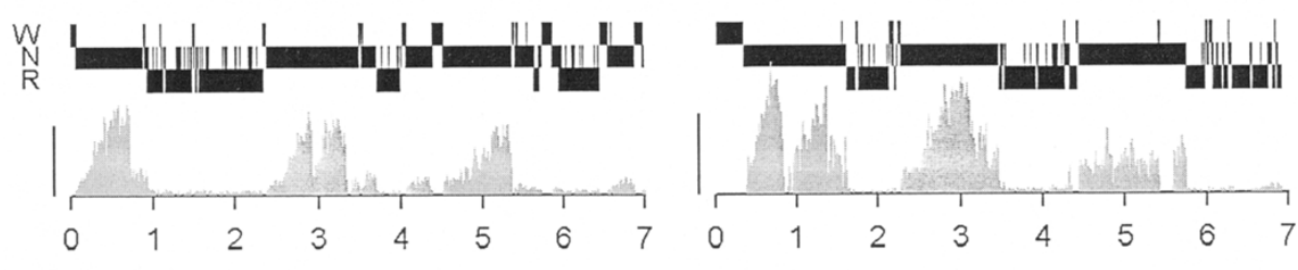

Hours after lights-out

Figure 1. Sleep profiles and EEG slow-wave activity (SWA, power within 0.75-4.5 Hz; C3-A2 derivation) of representative nights of patients PM and RH in baseline, during subchronic and chronic phenelzine treatment, and in the first night after withdrawal. W: wakefulness (including movement time [MT]). N: nonREM sleep (stages 1-4). R: REM sleep. The curves of SWA were smoothed by a 3-point moving average. Artifacts and data of W and MT were excluded. The calibration marks at the left of the $y$-axes of the bottom panels represent $10^{3} \mu \mathrm{V}^{2}$.

dicates that the suppression of REM sleep was not merely due to the absence of electrophysiological signs of this sleep state. Nevertheless, two weeks of rebound appear unlikely to be long enough to regain all REM sleep, which had been lost during the months of phenelzine treatment. It has previously been hypothesized that accumulated REM sleep pressure may be reduced and/or replaced by wakefulness (Endo et al. 1998; Horne 2000). The enhancement of TFA in waking during treatment and its weak inverse relationship with REM sleep is consistent with this view, and may be evidence for compensatory REM sleep mechanisms occurring during wakefulness.

Although the present data need to be interpreted cautiously because they were obtained in only a few pa- tients whose compliance could not be verified by determination of phenelzine blood plasma levels, the findings indicate that REM sleep reoccurs in a cyclic manner within sleep episodes after several months of absence during chronic MAOI treatment. This observation has implications for hypotheses regarding the regulation and function of REM sleep.

\section{ACKNOWLEDGMENTS}

We wish to dedicate this manuscript to Dr. J. Christian Gillin and thank him cordially for his generous support. We thank R. Wong, A. Schlosser and D. Sweat for their help with data collection, Drs. E. Raimo and B. Schnierow for clinical care, 
Dr. A. A. Borbély for lending us two PS-1 recording systems, and Drs. P. Achermann, C. Kopp and I. Tobler for comments on the manuscript. The study was supported by the Swiss National Science Foundation (823A-0566161), the National Institute of Mental Health (MH38738), the UCSD MHCRC (MH30914), the General Clinical Research Center (M01RR00827), the Department of Veterans Affairs, and the UCSD Fellowship in Psychopharmacology and Psychobiology.

\section{REFERENCES}

Akindele MO, Evans JI, Oswald I (1970): Mono-amine oxidase inhibitors, sleep and mood. Electroenceph clin Neurophysiol 29:47-56

Dement W (1960): The effects of dream deprivation. Science 131:1705-1707

Dunleavy DLF, Oswald I (1973): Phenelzine, mood response, and sleep. Arch Gen Psychiatry 28:353-356

Endo T, Roth C, Landolt HP, Werth E, Aeschbach D, Achermann P, Borbély AA (1998): Selective REM sleep depri- vation in humans: effects on sleep and sleep EEG. Am J Physiol 274:R1186-R1194

Horne JA (2000): REM sleep-by default? Neurosci Biobehav Rev 24:777-797

Landolt HP, Raimo EB, Schnierow BJ, Kelsoe JR, Rapaport MH, Gillin JC (2001): Sleep and sleep electroencephalogram in depressed patients treated with phenelzine. Arch Gen Psychiatry 58:268-276

Rechtschaffen A, Kales A (eds) (1968): A manual of standardized terminology, techniques and scoring system for sleep stages of human sleep. Washington, D.C.: Public Health Service, U.S. Government Printing Office

Wyatt RJ, Fram DH, Buchbinder R, Snyder F (1971a): Treatment of intractable narcolepsy with a monoamine oxidase inhibitor. New Engl J Med 285:987-991

Wyatt RJ, Fram DH, Kupfer DJ, Snyder F (1971b): Total prolonged drug-induced REM sleep suppression in anxious-depressed patients. Arch Gen Psychiatry 24:145-155 